Revista Complutense de Educación

ISSNe: 1988-2793

http://dx.doi.org/10.5209/RCED.56243

\title{
Agenda de trabajo del orientador. En centros de educación infantil y primaria
}

Autores: Celia Carrera, Teresa López, Paloma Matías y Consuelo Santamaría Editorial: Narcea

Año de publicación: 2016

$N^{a}$ de páginas: 213

ISBN: 978-84-277-2136-4

En esta obra, las autoras aúnan sus experiencias profesionales como miembros del mismo grupo de orientación, para elaborar una agenda de trabajo para los profesionales que acaban de incorporarse al mundo de la orientación educativa. Las autoras facilitan una serie de herramientas que son de gran ayuda para el orientador/a. Los principios básicos que les guían son la fijación de unas metas realistas, la toma de conciencia del papel del orientador colaborando en la construcción de soluciones compartidas, asumir que la tarea no es el rastreo de discapacidades o etiquetar al alumnado y la dedicación de tiempo a la formación permanente y al debate con otros profesionales.

El texto se divide en cinco capítulos: los dos primeros, hacen una síntesis de, en primer lugar, la introducción y ámbitos de intervención en la orientación educativa $\mathrm{y}$, en segundo lugar, la agenda que se desarrolla en los siguientes capítulos a través de un cronograma y una breve explicación de la forma en que se desarrolla la obra. Los dos siguientes capítulos hacen referencia a las actuaciones o tareas que se desarrollan, dividiéndolos en tareas que se desarrollan mes a mes y tareas a lo largo del curso. Finalmente, se aportan una serie de anexos para facilitar el desarrollo de las tareas o actuaciones.

El capítulo 1: La orientación educativa en las primeras etapas, se abre con la afirmación de que las primeras etapas educativas resultan fundamentales para el alumnado en cuanto al aprendizaje intelectual, la motivación y el hábito de estudio. A continuación, se detalla lo que las autoras consideran una orientación de calidad, que corresponde a una orientación que, ajuste la enseñanza y las ayudas a la gran diversidad del alumnado, establezca una base sólida para una buena colaboración familia-escuela, que sepa coordinarse con otros recursos comunitarios, que se anticipe a la aparición de dificultades de aprendizaje y detecte cuanto antes las necesidades para aportar los apoyos y adaptaciones necesarias.

Los ámbitos de intervención a los que se hace referencia en este capítulo son: asesoramiento a la institución, para las autoras el más complejo, puesto que es necesario ajustar el trabajo a otros profesionales, planificando y estructurando organizativamente el centro, realizando propuestas pedagógicas y sabiéndose adaptar a los diferentes contextos escolares y características de los centros; el apoyo al proceso de enseñanza y aprendizaje, resulta para las autoras el más visible y de mayor trabajo puesto que se refiere a la puesta en marcha y desarrollo de los planes 
a través de actuaciones y tareas en ámbitos muy variados pero advierten del peligro de enfocar la orientación de forma clínica o terapéutica; la colaboración familiaescuela, fundamentando que la educación del niño es una tarea de respeto profundo compartida por la familia y la escuela a lo que los orientadores pueden ayudar buscando acuerdos y compromisos concretos entre ellos, facilitando conocimiento para llevar a cabo cambios positivos y restaurando la confianza y comunicación entre ellos a través de las asociaciones de padres, grupos de familias de una etapa, ciclo o nivel, etc.; finalmente, el ámbito de colaboración con otros servicios e instituciones hace referencia a la coordinación con la red de servicios a través del conocimiento del contexto sociocultural, recursos sanitarios, culturales, sociales y de ocio y tiempo libre así como la atención orientadora al alumnado que hace uso de esos servicios por diferentes motivos como de la coordinación entre los servicios de orientación y profesorado cuando el alumnado cambia de centro.

En el segundo capítulo: La Agenda del Orientador, se hace referencia a una de las cuestiones más laboriosas del orientador, como es la organización del trabajo a lo largo del curso con una secuencia temporal clara. Se realiza una propuesta de distribución temporal de las actuaciones conformadas para un plan anual de orientación para poder elaborar una agenda de trabajo.

Las actuaciones se distribuyen en dos bloques, actuaciones mes a mes, haciendo referencia a aquellas actuaciones de todos los ámbitos que se desarrollan en un momento concreto desde el mes de septiembre hasta el mes de junio y, por otro lado, a las actuaciones a lo largo del curso, refiriéndose a las actuaciones que deben realizarse en momentos puntuales a lo largo de curso.

Finalmente, se explica la organización del desarrollo de cada actuación o tarea, que organizan en cuatro apartados: unas consideraciones previas, que recogen una base conceptual y principios pedagógicos que respaldan la actuación, un procedimiento, que recoge la secuencia de pasos o del proceso, unos documentos de apoyo como material práctico que apoya la actuación y unas claves para una buena práctica, recogiendo algunos principios o recomendaciones que las autoras desean transmitir para un buen hacer y orientación de calidad.

El tercer capítulo: Desarrollo de tareas o actuaciones mes a mes, recoge una serie de tareas que las autoras consideran que deben a hacerse en un momento específico del curso.

El cuarto capítulo: Desarrollo de tareas o Actuaciones a lo Largo del Curso (ALC), hace referencia a tareas a actuaciones que no se dan en un momento específico del curso, sino que responden a sucesos que van surgiendo a lo largo de éste de forma espontánea.

Se hace referencia a actuaciones de participación en claustros u otras estructuras de coordinación pedagógica, las evaluaciones psicopedagógicas, la colaboración en la intervención de problemas de conducta y el desarrollo de la acción tutorial, el asesoramiento de programas de mejora y la colaboración familia-escuela, la coordinación con otros profesionales y la organización de un banco de recursos.

Finalmente, en los Anexos, se adjunta numerosa documentación y material práctico para la puesta en marcha de algunas de las tareas o actuaciones, que, según las autoras, deben ser adaptados a cada realidad concreta.

En síntesis y sieguiendo a las autoras, la obra refleja una forma de entender la orientación educativa como un proceso de colaboración, reflexión conjunta y búsqueda de soluciones compartidas en todos los ámbitos de intervención pedagógica.

Estrella María Arranz Duarte Universidad Complutense de Madrid estrellaarranzduarte@ucm.es 